\title{
Chemotherapy-induced niche perturbs hematopoietic reconstitution in B-cell acute lymphoblastic leukemia
}

Chao Tang ${ }^{1}$, Ming-Hao Li ${ }^{1}$, Ya-Li Chen ${ }^{1}$, Hui-Ying Sun ${ }^{1}$, Sheng-Li Liư', Wei-Wei Zheng ${ }^{1}$, Meng-Yi Zhang ${ }^{1}$, Hui Li ${ }^{1}$, Wei Fu', Wen-Jun Zhang ${ }^{2}$, Ai-Bin Liang ${ }^{2}$, Zhong-Hua Tang ${ }^{1}$, Deng-Li Hong ${ }^{3}$, Bin-Bing S. Zhou ${ }^{1 *+}$ and Cai-Wen Duan ${ }^{1 *+}$

\begin{abstract}
Background: Considerable efforts have been devoted toward the uncovering of the molecular mechanisms underlying the maintenance of hematopoietic stem cells (HSCs) by the normal bone marrow (BM) niche. Previously, we demonstrated that a chemotherapy-induced niche, which is mainly composed of mesenchymal stem cells (MSCs), protects the residual B-cell acute lymphoblastic leukemia (B-ALL) cells from the insult of chemotherapeutic drugs. However, the roles of chemotherapy-induced niche on HSCs functions in B-ALL remain unclear.

Methods: We established an oncogenic N-MYC-driven B-ALL mouse model, which were subsequently treated with common chemotherapy drug cytarabine (Ara-C) and daunorubicin (DNR). After treatment, the structures of the BM niche were imaged by immunofluorescence staining. Then, the self-renewal and differentiation capability of the MSCs in the BM after Ara-C and DNR treatment were studied by ex vivo culture and gene expression analysis with RNA-seq and qRT-PCR. The effects of chemotherapy-induced niche on the hematopoietic reconstitution of HSCs were determined with series transplantation assay. Furthermore, the cell cycle, ROS level, mitochondrial membrane potential and cell apoptosis of HSCs were detected by flow cytometry.

Results: The MSCs, which is the main component of chemotherapy-induced BM niche, have decreased self-renewal capability and are prone to differentiate into adipocytes and chondrocytes. The results of gene expression analysis with RNA-seq showed that the MSCs have reduced levels of cytokines, including SCF, CXCL12, ANGPT1, VCAM1, and IL7. Furthermore, the chemotherapy-induced niche perturbed the hematopoietic reconstitution of HSCs in our N-MYC-driven B-ALL mouse model by promoting HSCs to enter cell cycle and increasing intracellular ROS levels and mitochondrial membrane potential of HSCs, which lead to the cell apoptosis of HSCs.
\end{abstract}

Conclusions: Chemotherapy-induced BM niche perturbs the hematopoietic reconstitution of HSCs by increasing intracellular ROS level and inducing cell apoptosis.

Keywords: Acute lymphoblastic leukemia; chemotherapy-induced niche, Hematopoietic reconstitution, Hematopoietic stem cells, Mesenchymal stem cells

\footnotetext{
*Correspondence: zhoubinbing@scmc.com.cn; duancaiwen@scmc.com.cn

${ }^{\dagger}$ Bin-Bing S. Zhou and Cai-Wen Duan contributed equally to this work. 1 Key Laboratory of Pediatric Hematology and Oncology Ministry of Health and Pediatric Translational Medicine Institute, Shanghai Children's Medical Center, Shanghai Collaborative Innovation Center for Translational Medicine and Department of Pharmacology and Chemical Biology, Shanghai Jiao Tong University School of medicine (SJTU-SM), Shanghai 200025, China Full list of author information is available at the end of the article
}

(c) The Author(s). 2018 Open Access This article is distributed under the terms of the Creative Commons Attribution 4.0 International License (http://creativecommons.org/licenses/by/4.0/), which permits unrestricted use, distribution, and reproduction in any medium, provided you give appropriate credit to the original author(s) and the source, provide a link to the Creative Commons license, and indicate if changes were made. The Creative Commons Public Domain Dedication waiver (http://creativecommons.org/publicdomain/zero/1.0/) applies to the data made available in this article, unless otherwise stated. 


\section{Background}

Hematopoiesis is a continuous process of orchestrated proliferation, self-renewal, and differentiation of hematopoietic stem cells (HSCs) in the bone marrow (BM) and followed by the egress of mature progeny into the circulating blood $[1,2]$. HSCs are the only cells in mammals capable of producing all blood cell lineages throughout life [3]. Chemotherapy may induce hematopoietic deficiency, which is potentially fatal and associated with $20 \%$ mortality in patients with acute leukemia or high-grade lymphoma $[4,5]$. Up to $30-40 \%$ of patients treated with several cycles of chemotherapy failed to mobilize HSCs, and this condition often precludes transplantation and compromises the chance of long-term survival $[6,7]$.

The BM niche is thought to maintain HSCs stemness while regulating HSCs maturation and differentiation $[8,9]$. Although the several genetic studies have provided mechanistic insights into the functions of $\mathrm{BM}$ niche cells, mainly MSCs, in the regulation of hematopoiesis during homeostasis [10,11], the effects of BM niche after stress or injury (such as chemotherapy) on HSCs remains unclear. Chemotherapy exhibits acute cytotoxic effects [12, 13]. In particular, patients that have received several rounds of chemotherapy frequently show irreversible chronic bone marrow damage that often leads to impaired hematopoietic reserve and function [6].

B-cell acute lymphoblastic leukemia (B-ALL) is one of the most common pediatric cancers. Although the treatment of pediatric leukemia has achieved great success owing to the improved regimes of chemotherapy and the five-year overall survival rates of B-ALL exceed $85 \%$ in children [14], the chance of relapse and impaired hematopoietic reconstitution after chemotherapy remain major challenges. In our previous study, we demonstrated that a chemotherapy-induced niche shielding residual B-ALL cells can be rebuilt by recruiting BM cells via cytokines secreted from B-ALL cells after chemotherapy [15]. However, whether the chemotherapy-induced niche affects the hematopoietic reconstitution of HSCs in B-ALL is still unknown. Here, we found that chemotherapy-induced niche perturbed the hematopoietic reconstitution in our B-ALL mouse model by dysregulating intracellular ROS levels and inducing cell apoptosis of HSCs. These results rovide a mechanistic explanation for how chemotherapy impairs normal hematopoietic homeostasis, which is important for the consideration of BM transplantation in patients with B-ALL after they undergo chemotherapy.

\section{Methods}

Animals and B-ALL mouse model

Adult C57/B6 mice (8-12 weeks old) were obtained from Shanghai SLAC Laboratory Animal Co., Ltd.
CD45.1/2 congenic strains were bred in our facilities. All animal experiments were approved and monitored by the Shanghai Children's Medical Center Animal Care and Use Committee. An N-MYC-driven B-ALL mouse model was constructed according to the methods described in previous studies [16]. In all experiments, mice were euthanized by $\mathrm{CO}_{2}$ asphyxiation. No randomization or blinding was used for the allocation of the experimental groups.

\section{Chemotherapeutic drug treatment}

For the assessment of the role of chemotherapy in BM transplantation, mice were injected i.p. with Ara-C ( $1 \mathrm{~g} / \mathrm{kg}$ body weight) or DNR ( $15 \mathrm{mg} / \mathrm{kg}$ body weight) up to 2 days before they were euthanized for analysis.

\section{Immunofluorescence imaging}

Whole-mount tissues and the frozen sections of long bones were prepared as follows: femoral or tibial bones were perfusion-fixed and then post-fixed for $30 \mathrm{~min}$ in $4 \%$ paraformaldehyde (PFA), incubated in $20 \%$ sucrose for cryoprotection, and finally embedded in EBM (embedding buffer). For whole-mount staining, bones were shaved on a cryostat until the bone marrow cavity was fully exposed. Bones were carefully harvested from the melting EBM. Frozen sections were prepared according to the nature protocol [17]. Sections were permeated in $0.1 \%$ Triton-X100 dissolved in $1 \times$ PBS for $30 \mathrm{~min}$. Then, the sections were blocked in $20 \%$ normal goat serum for $1 \mathrm{~h}$. Primary antibodies were diluted and incubated with fixed cells overnight at $4{ }^{\circ} \mathrm{C}$. Next day, the sections were washed for three times in PBS and then incubated with the matching secondary antibodies for $1 \mathrm{~h}$ at room temperature. DAPI were then added to the stain nucleus for $10 \mathrm{~min}$. After washing in PBS, the sections were mounted with antifade fluorescence mounting medium. Images were acquired on a Leica SP8 confocal microscope.

\section{Flow cytometry analysis}

Fluorochrome-conjugated or biotinylated $\mathrm{mAbs}$ specific to mouse CD45 (clone 30-F11), CD45.1 (clone A20), Ter119 (clone Ter-119), c-Kit (clone 2B8), Sca-1 (clone D7), CD41 (clone MWReg30), CD150 (clone TC15-12F12.2), hematopoietic lineage cocktail, and corresponding isotype controls were purchased from eBioscience. CD31 (clone MEC13.3) and CD48 (clone HM48-1) were from purchased from BioLegend. Cells were analyzed on a LSR II Flow Cytometer (Becton Dickinson). 


\section{Cell culture and differentiation}

For the clonal sphere formation, cells were plated at clonal density $\left(<500\right.$ cells $\left./ \mathrm{cm}^{2}\right)$ or by single cell sorting into ultralow adherent plates as previously described [18]. Cells were kept at $37{ }^{\circ} \mathrm{C}$ and with $5 \% \mathrm{CO}_{2}$ in a water-jacketed incubator and left untouched for 1 week for the prevention of cell aggregation. One-half medium changes were performed weekly. All spheres in each well were counted at day 9, and results were expressed as a percentage of plated cells. For osteogenic, adipogenic, and chondrogenic differentiation, mouse CD45 $5^{-}$Ter $119^{-}$ $\mathrm{CD}^{-} 1^{-} \mathrm{PDGFR}^{+} \mathrm{CD} 51^{+}$cells were treated with StemXVivo Osteogenic, Adipogenic, or Chondrogenic mouse specific differentiation media according to the manufacturer's instructions (R\&D Systems). All cultures were maintained with $5 \% \mathrm{CO}_{2}$ in a water-jacketed incubator at $37{ }^{\circ} \mathrm{C}$. Osteogenic differentiation indicated by mineralization of extracellular matrix and calcium deposits was revealed by Alizarin Red S staining. Cells were fixed with $4 \%$ paraformaldehyde (PFA) for $30 \mathrm{~min}$. After rinsing in distilled water, the cells were stained with $40 \mathrm{mM}$ Alizarin Red S (Sigma-Aldrich) solution at $\mathrm{pH} 4.2$, rinsed in distilled water, and finally washed in Tris-buffered saline for $15 \mathrm{~min}$ for the removal of nonspecific staining. Adipocytes were identified by the typical production of lipid droplets and Oil Red staining. Chondrocytes were revealed by Alcian Blue staining, which detects the synthesis of glycosaminoglycans. The cells were fixed with $4 \%$ PFA for 60 min, embedded in paraffin, and sectioned. The sections were incubated with $0.5 \%$ Alcian Blue (Sigma-Aldrich) in distilled water for $15 \mathrm{~min}$. To remove nonspecific staining, sections were rinsed three times with running water (5 min each).

\section{Hematopoietic transplantation assay}

Competitive repopulation assays were performed using the CD45.1/CD45.2 congenic system. Equivalent volumes of bone marrow cells were collected from an adult mouse. LSKs were transplanted into lethally irradiated CD45.1 recipients with $0.2 \times 10^{5}$ competitor CD45.1 cells. The CD45.1/CD45.2 chimaerism of recipients' blood was analyzed up to 4 months after transplantation.

\section{RNA extraction and quantitative real-time PCR}

Briefly, $500 \mathrm{ng}$ of total RNA was used for cDNA synthesis. Random primers (Qiangen, GM) were used, and all the primer pairs were designed to overlap one intron such that the amplification of genomic DNA is prevented. Q-PCR was sequentially conducted by using SYBR Green PCR master Mix (Takara, JP) and a real-time PCR thermocycler (Angelent), and data were analyzed with the supplied ABI software. Q-PCR was conducted with the following primers:

\begin{tabular}{|c|c|c|}
\hline & Forward $\left(5^{\prime}-3^{\prime}\right)$ & Reverse (5'-3') \\
\hline Actin & TCTGGCACCACACCTTCTACAAT & $\begin{array}{l}\text { TGGGGTGTTGAAGGTCTCA } \\
\text { AA }\end{array}$ \\
\hline $\mathrm{Cfd}$ & $\begin{array}{l}\text { TGCATCAACTCAGAGTGTC } \\
\text { AATCA }\end{array}$ & TGCGCAGATTGCAGGTTGT \\
\hline Gpnmb & CCCCAAGCACAGACTTTTGAG & GCTTTCTGCATCTCCAGCCT \\
\hline Ogn & ACCATAACGACCTGGAATCTGT & AACGAGTGTCATTAGCCTTGC \\
\hline Sp7 & ATGGCGTCCTCTCTGCTTGA & GAAGGGTGGGTAGTCATTTG \\
\hline Acan & CACGCTACACCCTGGACTTTG & $\begin{array}{l}\text { CCATCTCCTCAGCGAAGCA } \\
\text { GT }\end{array}$ \\
\hline Col11a2 & TGGCACTCCTGGTCCAGAAG & GCCGGGCTTTCCTGCTA \\
\hline
\end{tabular}

\section{RNA-sequencing (RNA-seq) analysis}

The cells were sorted into RLT buffer (Qiagen RNAeasy Plus Micro kit), and RNA was purified according to the manufacturer's instructions. Sequencing libraries were prepared from $10 \mathrm{ng}$ to $100 \mathrm{ng}$ total RNA by using the TruSeq RNA Sample Preparation Kit v2 (Illumina). All the samples were sequenced by Illimina plantform Hiseq $\mathrm{X}$ Ten, and all the obtained sequences had a $100 \mathrm{bp}$-long pair end. Raw sequenced reads were mapped to mouse reference genome hg19 by STAR (version 2.3.0). Fragments per kilobase of an exon model per illion (FPKM) mapped fragments for each gene was generated by cufflinks software. The number of reads mapped to each gene was counted by HTSeq. Differential expressed genes were generated by DESeq2 $R$ package. Genes with $\mathrm{p}$ of $<0.01$ and $\mid \log 2$ (fold change) $\mid$ of $>2$ were defined as differential expressed genes between each groups.

\section{Cytokine array}

A commercially available cytokine array of 80 cytokine proteins (Mouse Cytokine Antibody Array C1000; RayBiotech) was used to evaluate cytokine production. Briefly, supernatants obtained from the BM of the Ctrl $+\mathrm{A} 2 \mathrm{D}$ and B-ALL+A2D mice were tested for cytokine levels following the manufacturer's instructions. The intensity of each dot was quantified using ImageJ software from BioRad, and the signals were normalized relative to the background. Array map is showen in the following tables: 


\begin{tabular}{|c|c|c|c|c|c|c|c|c|c|c|c|c|c|c|}
\hline & $A$ & B & $C$ & $\mathrm{D}$ & $E$ & $\mathrm{~F}$ & G & $\mathrm{H}$ & I & J & K & $L$ & $M$ & $N$ \\
\hline 1 & Pos & Pos & Neg & Neg & Blank & $A \times l$ & Cxcl13 & $\mathrm{Cd} 30 \mathrm{~L}$ & $\mathrm{Cd} 30$ & $\mathrm{Cd} 40$ & $\mathrm{Crg}-2$ & $\mathrm{Ccl} 27$ & Cxcl16 & Col11 \\
\hline \multicolumn{15}{|l|}{2} \\
\hline 3 & $\mathrm{CCl} 24$ & FasL & $\mathrm{C} \times 2 \mathrm{Cl} 1$ & Gcsf & Gm-csf & Ifn- $\gamma$ & lgfbp3 & lgfbp5 & lgfbp6 & $\| 1-a$ & $\| 1-\beta$ & $\| 2$ & 113 & $\| 3 R \beta$ \\
\hline \multicolumn{15}{|l|}{4} \\
\hline 5 & $\| 4$ & $\| 5$ & 116 & 119 & $\| 10$ & II12 p40/p70 & II12 p70 & $\| 13$ & $\| 17 a$ & Cxcl1 & LepR & Leptin & Lix & $\mathrm{Cd} 62 \mathrm{~L}$ \\
\hline \multicolumn{15}{|l|}{6} \\
\hline 7 & Xcl1 & $\mathrm{Ccl} 2$ & Mcp5 & Mcsf & Cxcl9 & $\mathrm{Ccl} 3$ & Mip1r & Mip2 & Ccl19 & $\mathrm{Ccl} 20$ & Cxcl4 & P-Sel & $\mathrm{Ccl} 5$ & SCF \\
\hline \multicolumn{15}{|l|}{8} \\
\hline 9 & Cxcl12 & Col17 & $\mathrm{Ccl} 1$ & $\mathrm{Ccl} 25$ & Timp1 & Tnfa & TnfRI & TnfRII & Tpo & Vcam1 & Vegfa & Blank & Blank & Pos \\
\hline 10 & & & & & & & & & & & & & & \\
\hline
\end{tabular}

\begin{tabular}{|c|c|c|c|c|c|c|c|c|c|c|c|c|}
\hline & $A$ & $B$ & $C$ & $\mathrm{D}$ & $E$ & $F$ & $G$ & $\mathrm{H}$ & I & J & K & $\mathrm{L}$ \\
\hline 1 & Pos & Pos & Neg & Neg & Blank & bFgf & $\mathrm{Cd} 26$ & Dtk & E-Sel & FcyRllb & Flt3L & GitR \\
\hline \multicolumn{13}{|l|}{2} \\
\hline 3 & $\mathrm{HgfR}$ & Icam1 & lgfbp2 & $\operatorname{lgf1}$ & $\lg f 2$ & $\| 15$ & II17Rb & 117 & Cxcl11 & Cxcl15 & $\mathrm{Ccl} 22$ & Mmp2 \\
\hline \multicolumn{13}{|l|}{4} \\
\hline 5 & Mmp3 & Opn & Opg & Pro-mmp9 & Resistin & Shh-N & Cxcl7 & Timp2 & Trance & Troy & Tslp & VegfR1 \\
\hline \multicolumn{13}{|l|}{6} \\
\hline 7 & VegfR2 & VegfR3 & Vegfd & Blank & Blank & Blank & Blank & Blank & Blank & Blank & Blank & Pos \\
\hline 8 & & & & & & & & & & & & \\
\hline
\end{tabular}

\section{Cell cycle experiment for HSCs}

Cell cycle analysis for HSCs was performed as described previously [19]. Bone marrow was harvested by flushing the bone with $1 \mathrm{~mL}$ of ice-cold PBS. Then, the red blood cells were lysed once for $5 \mathrm{~min}$ at room temperature in ACK buffer and then washed once in ice-cold PBS. The cells were subsequently counted with a hemocytometer. For flow cytometry, the red blood cells were lysed three times for $5 \mathrm{~min}$ at room temperature in ACK buffer and washed once in ice-cold PBS and counted in a hemocytometer. For $\mathrm{Lin}^{-} \mathrm{Sca} 1^{+} \mathrm{C}-\mathrm{Kit}^{+} \mathrm{CD} 48^{-} \mathrm{CD} 150^{+}$detection, $10^{6}$ cells were stained with HSCs surface markers $30 \mathrm{~min}$ at $4{ }^{\circ} \mathrm{C}$, then fixed in $4 \%$ PFA in PBS for $30 \mathrm{~min}$ at $4{ }^{\circ} \mathrm{C}$, washed with PBS, permeabilized with $0.1 \%$ Triton $\mathrm{X}-100$ in PBS, and finally stained with anti-ki67-FITC antibody (ebioscience) and DAPI (Life Technologies) at $20 \mathrm{mg} / \mathrm{mL}$ for $30 \mathrm{~min}$ at room temperature. The cells were washed with washing buffer and analyzed with LSRII flow cytometer (Becton Dickinson).

\section{ROS staining}

For the ROS staining of the HSCs, 5 million cells were stained with HSCs cell surface antibodies as described above. The stained cells were then washed and stained with H2-DCFDA (Life Technologies) for $30 \mathrm{~min}$ at $37^{\circ} \mathrm{C}$ in $\mathrm{Ca}^{2+}$ and $\mathrm{Mg}^{2+}$ free HBSS $+2 \%$ HIBS $+50 \mu \mathrm{M}$ verapamil (Sigma-Aldrich). After washing, the cells were analyzed by flow cytometry.

\section{Apoptosis analysis}

Apoptosis was measured by staining freshly harvested bone marrow cells (at least 1 million cells) with $\mathrm{Lin}^{-} \mathrm{Scal}^{+} \mathrm{C}-\mathrm{K}-$ $\mathrm{it}^{+} \mathrm{CD} 48^{-} \mathrm{CD} 150^{+}$for HSCs, followed by Annexin-V-PE and 7-AAD staining (BD Bioscience). After washing, the cells were analyzed by flow cytometry immediately.

\section{Mitochondrial membrane potential analysis}

Total numbers of cells from bone marrow were stained with HSC markers and then resuspended in $1 \mathrm{ml}$ of PBS. Then cells were incubated with $50 \mathrm{nM}$ DiIC1(5) (Invitrogen) at $37{ }^{\circ} \mathrm{C}$ for $15-30 \mathrm{~min}$. After washing, the cells were analyzed by flow cytometry immediately.

\section{Statistical analysis}

All data are presented as the mean $\pm \mathrm{SD}$ of at least three separate experiments. Statistical analysis was performed 


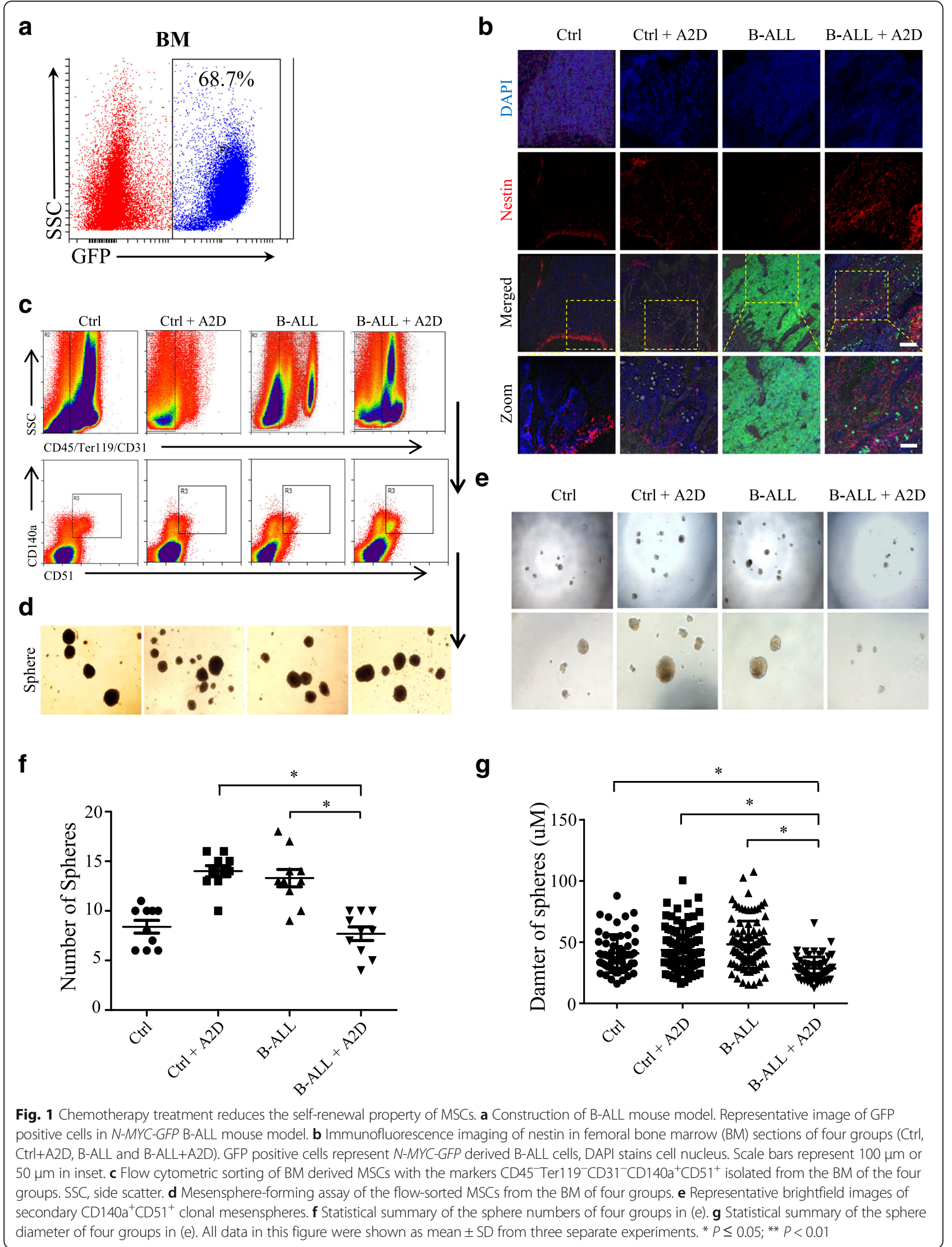




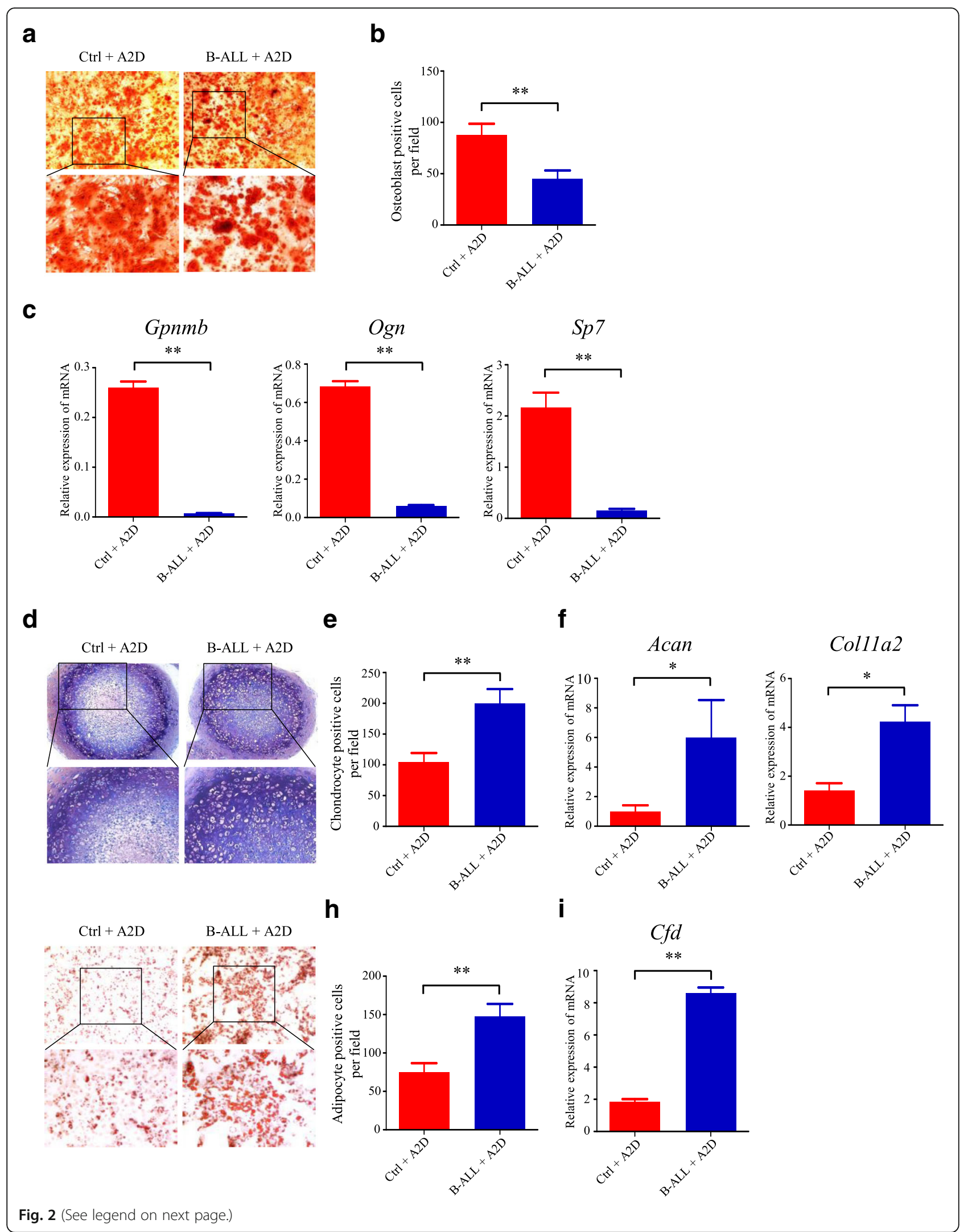


(See figure on previous page.)

Fig. 2 Chemotherapy-induced MSCs are prone to differentiate into adipocytes and chondrocytes. a Osteogenic differentiation of MSCs derived from Ctrl+A2D and B-ALL+A2D by Alizarin Red S staining. b Statistical summary of osteoblast number per field in (a). c The osteoblastic differentiation markers (Gpnmb, Ogn and Sp7) were evaluated by qRT-PCR. $\mathbf{d}$ Chondrogenic differentiation of MSCs derived from Ctrl+A2D and B-ALL+A2D by Toluidine Blue staining. e Statistical summary of chondrogenic cell number per field in (d). $\mathbf{f}$ The chondrogenic differentiation markers (Acan and Co/1 1a2) were evaluated by qRT-PCR. $\mathbf{g}$ Adipogenic differentiation of MSCs derived from Ctrl+A2D and B-ALL+A2D by Oil Red staining. $\mathbf{h}$ Statistical summary of adipogenic cell number per field in $(\mathrm{g})$. $\mathbf{i}$ The chondrogenic differentiation markers (Cfd) were evaluated by qRT-PCR. All data in this figure were shown as mean \pm SD from three separate experiments. ${ }^{*} P \leq 0.05 ; * * 0<0.01$

using one-way ANOVA followed by Dunnett's multiple comparison tests. Comparisons between two groups were analyzed using the Student's t-test. A $p$ value of less than 0.05 was statistically significant.

\section{Results}

\section{Chemotherapy treatment reduces the self-renewal property of MSCs}

To study the morphological structure of BM niche in B-ALL treated with chemotherapeutic drugs, we established the $N-M Y C$ driven B-ALL mouse model as reported previously [16] (Fig. 1a). Then, we treated the B-ALL mice i.p. with cytarabine (Ara-C) in vivo for two constitutive days (A2D). Consistent with our previous results [15], the cell numbers of Nestin $^{+}$mesenchymal stem cells (Nes-MSCs) increased dramatically in the B-ALL+A2D group compared with those of the other groups (Ctrl, B-ALL and Ctrl+A2D), as indicated by whole-mount immunofluorescence staining (Fig. 1b). To check whether the properties of the Nes-MSCs were alternated under the stress of chemotherapeutic agents, we sorted the BM-derived MSCs subset out of the total stromal cells $\left(\mathrm{CD} 45^{-} \mathrm{Ter} 119^{-} \mathrm{CD} 31^{-}\right)$from the four groups on the basis of the MSC specific markers $\mathrm{CD}_{140 \mathrm{a}^{+}}$and CD51 ${ }^{+}$[18] (Fig. 1c). We found that all of the MSCs subset of the four groups is capable of forming replatable mesenspheres in the conditional medium. After dissociation, these MSCs spheres passaged and formed secondary spheres as well, demonstrating the in vitro self-renewal capacity of the MSCs (Fig. 1d). To accurately compare the self-renewal capability of MSCs between these four groups, we dissociated the spheres into single cells and divided the cells into 100 cells per well in 96-wells plates. Interestingly, the capability of sphere-forming of secondary generation showed significant difference between B-ALL+A2D and other groups (Fig. 1e). Therefore, we calculated the numbers and diameter of spheres between four groups and found that the B-ALL+A2D group has dramatically decreased sphere numbers and sphere diameter (Fig. 1f-g), suggesting that chemotherapy treatment remarkably reduces the self-renewal property of MSCs in BM.

\section{Chemotherapy-induced MSCs are prone to differentiate into adipocytes and chondrocytes}

To further characterize the multilineage differentiation potentials of MSCs under the stress of chemotherapeutic agents, we plated MSCs spheres under in vitro mesenchymal lineage differentiation conditions. Multilineage differentiation was determined by specific staining and morphological and histochemical characterization of mature osteoblastic, chondrocytic, and adipocytic lineage phenotypes after $>30$ days of culture. Under conditions optimal for osteoblastic differentiation, clonal mesenspheres from the B-ALL+A2D group differentiated into less alizarin red stain positive osteoblast than that from the $\mathrm{Ctr}+\mathrm{A} 2 \mathrm{D}$ (Fig. 2a and b). Furthermore, we measured the gene expression levels of osteoblastic differentiation markers by qRT-PCR and found that the expression levels of Gpnmb, Ogn, and $S p 7$ genes were significantly decreased in the MSCs of the B-ALL+A2D group (Fig. 2c). This result demonstrated the impaired potential for osteoblastic differentiation. We also compared the chondrocytic differentiation by culturing MSCs with conditional medium and showed that the clonal mesenspheres of B-ALL+A2D group preferably differentiated into chondrocyte (Fig. $2 \mathrm{~d}$ and e). The result was subsequently confirmed by the gene expression analysis of the chondrocyte markers Acan and Col11a2 genes (Fig. 2f). We also found that the MSCs of B-ALL $+\mathrm{A} 2 \mathrm{D}$ group are prone to differentiate into adipocytes, as demonstrated by oil red staining (Fig. $2 \mathrm{~g}$ and $\mathrm{h}$ ) and gene expression analysis of adipocyte markers (Fig. 2i). Overall, these data demonstrate that the chemotherapyinduced MSCs in B-ALL have alternated differentiation potentials and may differentiate into adipocytes and chondrocytes.

\section{Chemotherapy-induced MSCs have reduced levels of HSC- maintaining cytokines}

To systematically characterize the potential differences between the four groups of chemotherapy-induced MSCs, we performed whole-genome RNA expression profile analysis with the MSCs of Ctrl, B-ALL, Ctrl $+\mathrm{A} 2 \mathrm{D}$, and B-ALL+A2D by total RNA sequencing (RNA-seq). The comparison results among the transcriptome profiles revealed the enrichment of several 
a

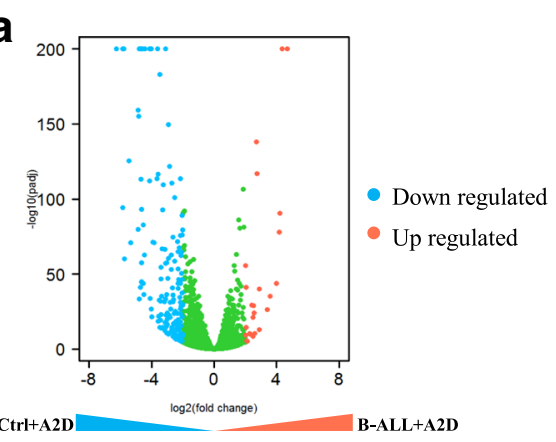

C

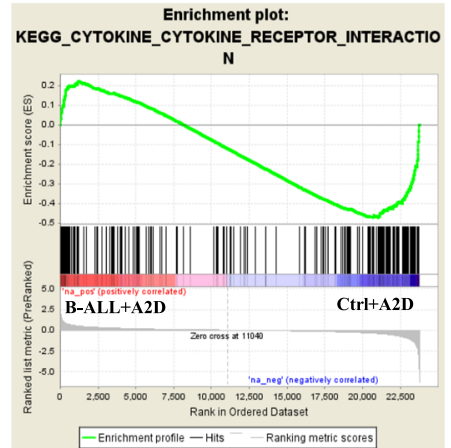

e
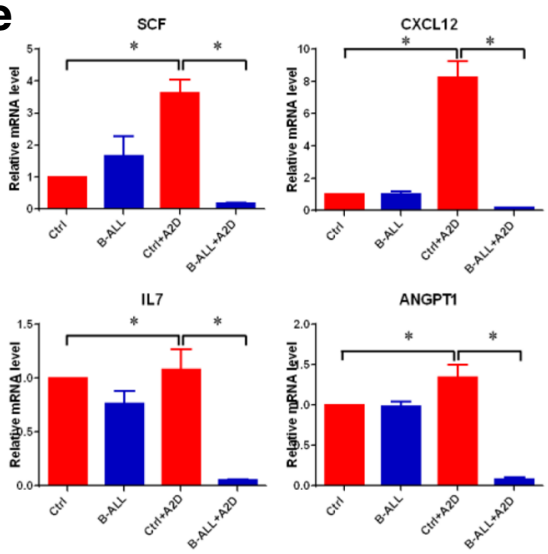

$\mathbf{f}$

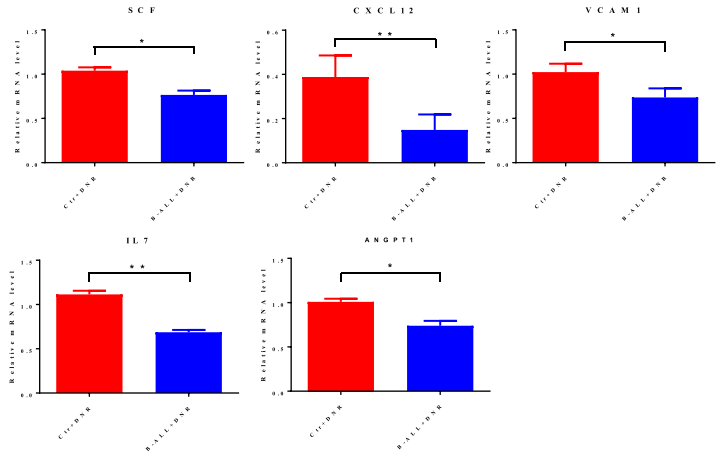

b

d

\section{KEGG pathway (B-ALL+A2D vs Ctrl+A2D)}

Cytokine-cytokine receptor interaction

Transcriptional misregulation in cancer Jak-STAT signaling pathway

Cell adhesion molecules (CAMs)

Fat digestion and absorption

Hematopoietic cell lineage

ECM-receptor interaction

Pancreatic secretion

Renin-angiotensin system
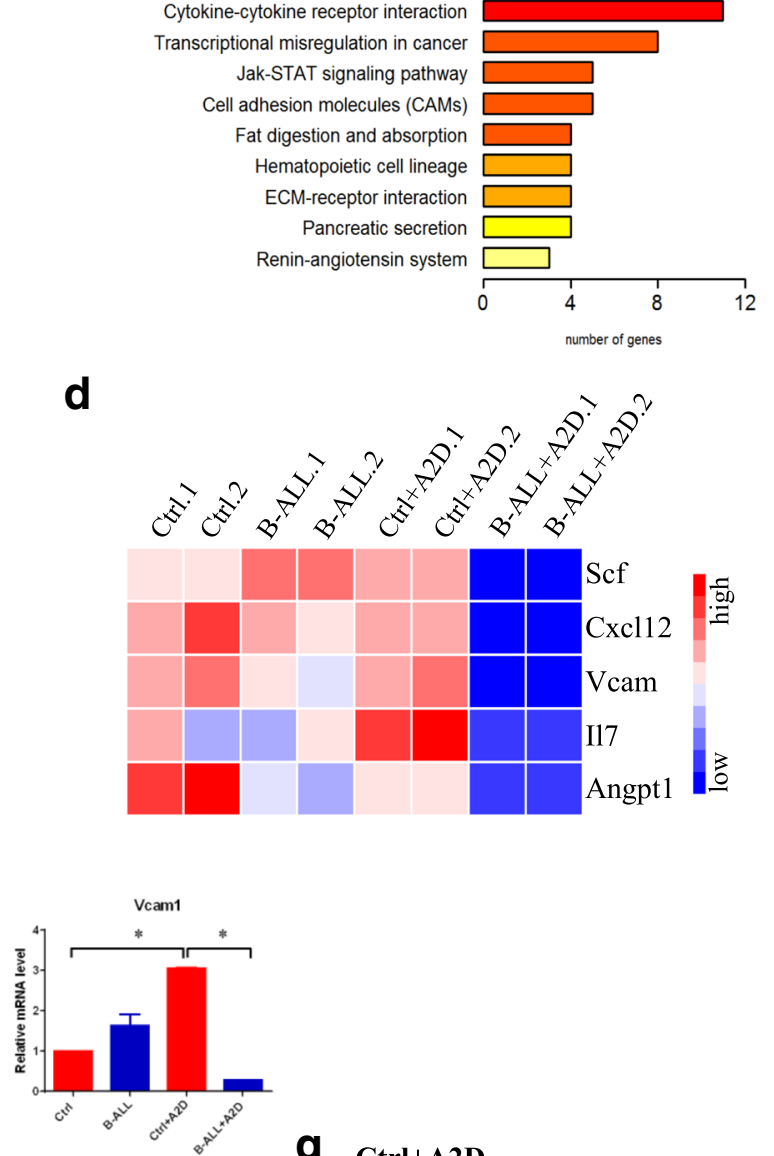

\section{Ctrl+A2D}

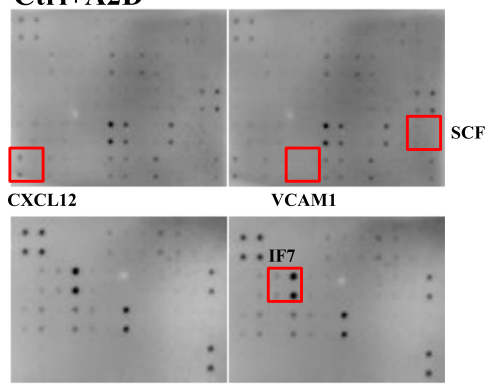

B-ALL+A2D

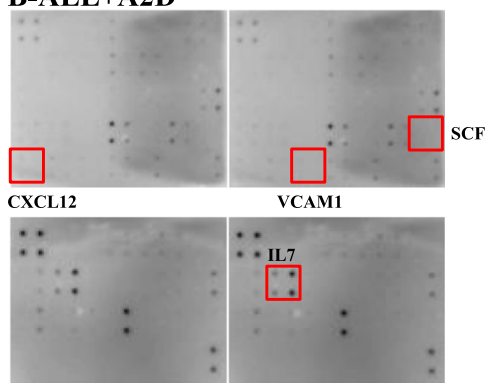

Fig. 3 (See legend on next page.) 
(See figure on previous page.)

Fig. 3 Chemotherapy-induced MSCs have reduced levels of HSC-maintaining cytokines. a RNA-seq analysis of differentially expressed genes upregulated and downregulated more than twofold in MSCs of Ctrl+A2D and B-ALL+A2D. Blue dots represent down-regulated genes and red dots represent up-regulated genes. $\mathbf{b}$ Differentially expressed pathways. B-ALL+A2D versus Ctrl+A2D. $\mathbf{c}$ Enrichment genes analysis of B-ALL+A2D and Ctrl+A2D. $\mathbf{d}$ Heat map analysis of SCF, CXCL12, VCAM1, IL7 and ANGPT1 from RNA-seq data. e The expression of SCF, CXCL12, VCAM1, IL7 and ANGPT1 in MSCs of Ctrl+A2D and B-ALL+A2D was evaluated by qRT-PCR. $\mathbf{f}$ The expression levels of SCF, CXCL12, VCAM1, IL7 and ANGPT1 in MSCs of Ctrl+DNR and B-ALL+DNR was evaluated by qRT-PCR. $\mathbf{g}$ The expression levels of SCF, CXCL12, VCAM1 and IL7 in bone marrow of Ctrl + DNR and B-ALL+DNR was detected by cytokine array. All data in this figure were shown as mean \pm SD from three separate experiments. * $P \leq 0.05 ; * * 0.01$

molecular pathways, such as cytokine-receptor interactions and transcriptional misregulation in cancer, in the B-ALL+A2D group (Fig. 3a-c). As cytokine-receptor interactions are crucial to the maintenance of HSCs through the regulation of the self-renewal and differentiation properties, we analyzed the gene expression of cytokines related to the maintenance of HSCs. Indeed, the MSCs of the B-ALL+A2D group showed significantly decreased gene expression levels of those cytokines, including SCF; cytokines CXCL12, ANGPT-1, and IL7; and vascular cell adhesion molecule-1 (VCAM-1) (Fig. 3d-e). Consistently, those five cytokines were downregulated in the MSCs of B-ALL after DNR treatment (Fig. 3f). As detected by cytokine array, the protein levels of three cytokines (SCF, CXCL12 and IL7) were also decreased in bone marrow of the B-ALL+A2D group compared with those of the Ctrl +A2D group (Fig. 3g). These cytokines regulate HSC maintenance and attraction in the bone marrow [8], suggesting that decreased cytokines expression of MSCs by chemotherapy can disrupt the functions of HSCs and compromise the hematopoietic reconstitution of HSCs.

\section{Chemotherapy-induced niche perturbs hematopoietic reconstitution of HSCs}

Since chemotherapy reduce the expression of HSC-maintaining cytokines in MSCs, we examined the effect of chemotherapy-induced niche on the hematopoietic reconstitution of HSCs with competitive reconstitution assays in two transplantation strategies. First, we sorted the HSCs ( $\mathrm{Lin}^{-} \mathrm{Sca}-1^{+} \mathrm{C}-\mathrm{Kit}^{-}$, LSK cells) from the Ctrl+A2D and B-ALL+A2D mice and transplanted them into recipients (CD45.1 mice) with equal numbers of cells (Fig. 4a). The transplantation ratio was analyzed every 4 weeks. The percentage of CD45.2 donor-derived cells from the B-ALL + A2D group was significantly decreased in the first and secondary transplantation (Fig. 4b-c), suggesting the decreased hematopoietic reconstitution capability of HSCs from the B-ALL+A2D group.

To rule out the possibility that the deficiency in the hematopoietic reconstitution of HSCs is caused by the long-term suppression from leukemic cells, we injected normal LSK-tomato ${ }^{+}$cells into Ctrl+A2D and B-ALL
$+\mathrm{A} 2 \mathrm{D}$ mice and then sorted the tomato ${ }^{+}$cells from the chemotherapy-induced BM with flow cytometry in $72 \mathrm{~h}$. The sorted tomato ${ }^{+}$cells were then transplanted into the recipient mice (CD45.2) (Fig. 4d). The results showed that the percentage of tomato ${ }^{+}$population from the B-ALL+A2D group decreased dramatically in the first transplantation than those from the Ctrl+A2D (Fig. 4e). Engraftment from the B-ALL+A2D group also showed a remarkable difference in the secondary transplantation (Fig. 4f). Additionally, we analyzed the lineage differentiation of HSCs and found that the HSCs from the B-ALL $+\mathrm{A} 2 \mathrm{D}$ group preferentially differentiated into megakaryocytes (Fig. 4g). These results showed that the hematopoietic reconstitution of the HSCs in the B-ALL + A2D group was compromised, at least in part, because of the dramatically decreased HSCs-maintaining cytokines in the chemotherapy-induced niche.

\section{Chemotherapy-induced niche increases the ROS levels of HSCs and induces cell apoptosis}

To get the insights of the perturbed hematopoietic reconstitution of HSCs, we studied the HSCs in the Ctrl $+\mathrm{A} 2 \mathrm{D}$ and B-ALL+A2D groups with the markers of Lineage ${ }^{-} \mathrm{sca}-1^{+} \mathrm{c}-\mathrm{kit}^{+} \mathrm{CD} 48^{-} \mathrm{CD} 150^{+}$as previously reported [20] (Fig. 5a). Firstly, we stained these HSCs with anti-ki67 antibody and DAPI and used flow cytometry to check the cell cycle of the HSCs from the two groups. The flow cytometry results showed that the percentage of HSCs in the $G_{0}$ phase of the B-ALL+A2D group was dramatically decreased than those of the Ctrl+A2D group (Fig. 5b and c). Next, we also compared the cell apoptosis of HSCs in the Ctrl+A2D and B-ALL+A2D groups with staining of annexin $\mathrm{V}$. The results demonstrated that the apoptotic cells in the B-ALL+A2D group drastically increased than those of the Ctrl+A2D group (Fig. 5d and e). Apoptosis was also significantly increased in the HSCs of B-ALL after DNR treatment (Additional file 1: Figure S1a and b). Together, these results suggest that the chemotherapy-induced niche perturbed the hematopoietic reconstitution of HSCs in B-ALL mouse model by promoting HSCs to enter cell cycle and inducing cell apoptosis.

Since oxidative stress is the result of an overabundance of cellular reactive oxygen species (ROS) accumulation 
a

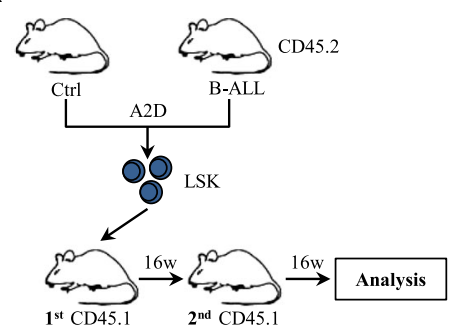

b

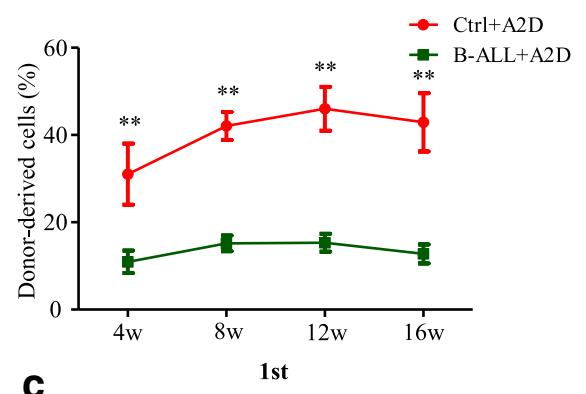

C

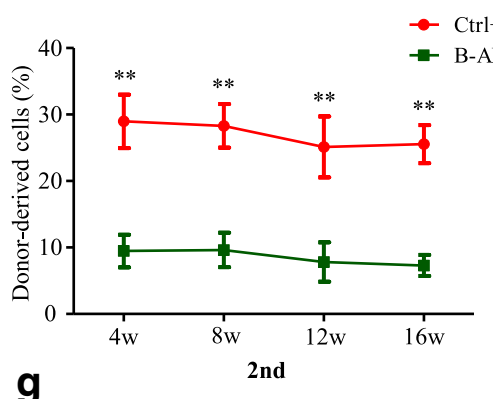

d

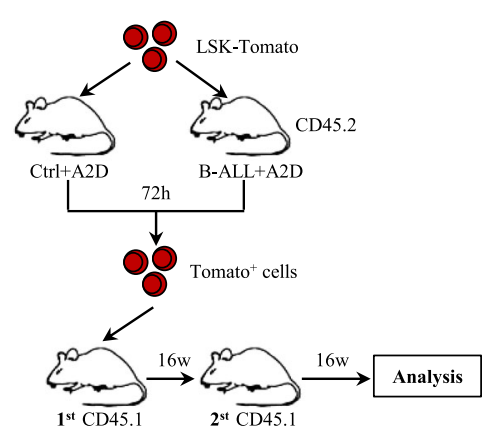

e

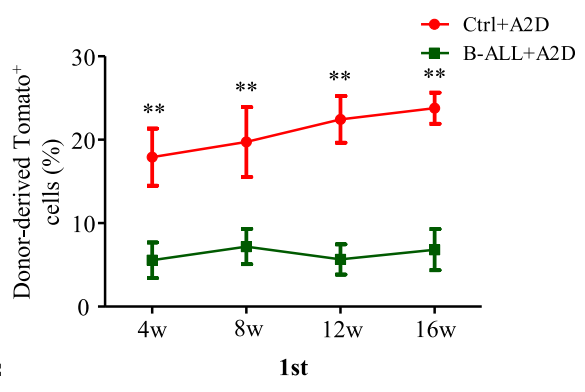

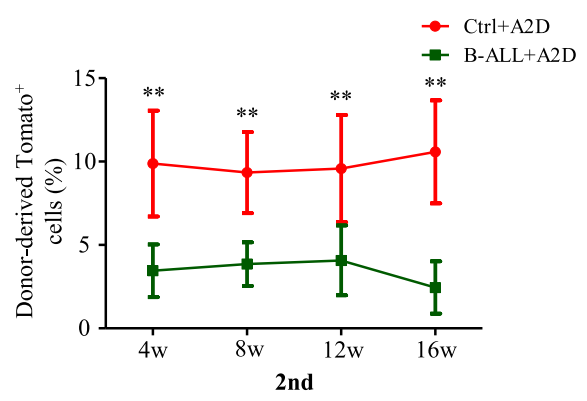

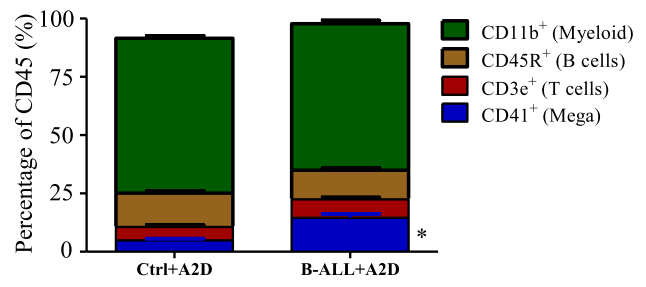

Fig. 4 Chemotherapy-induced niche perturbs hematopoietic reconstitution of HSCs. a Experimental design to determine the hematopoietic reconstitution of LSK cells isolated from Ctrl+A2D and B-ALL+A2D. b Percentages of donor-derived cells after first competitive reconstitution. $n=5-6$ mice per group. $\mathbf{c}$ Percentages of donor-derived cells after second transplantation. $n=5$ mice per group. $\mathbf{d}$ Experimental design to determine the hematopoietic reconstitution of normal LSK-tomato ${ }^{+}$cells isolated from the chemotherapy-induced niche of Ctrl+A2D and B-ALL + A2D. e Percentages of tomato ${ }^{+}$cells after first competitive reconstitution. $n=5$ mice per group. $\mathbf{f}$ Percentages of donor-derived cells after second transplantation. $n=5$ mice per group. $\mathbf{g}$ Flow cytometric analysis of lineage differentiation of LSK in (b). All data in this figure were shown as mean \pm SD from three separate experiments. * $P \leq 0.05 ;{ }^{*} P<0.01$

formed by the partial reduction of oxygen or a defect in the antioxidant protection mechanism [21]. High intracellular levels of ROS have been showed to limit the lifespan of HSCs and accelerate their differentiation and exhaustion [22]. We speculated that the cell cycle proliferation and increased cell apoptosis of HSCs in the B-ALL+A2D group might be caused by the increased intercellular ROS. To test our inferences, we analyzed the intercellular ROS levels of the HSCs in the Ctrl $+\mathrm{A} 2 \mathrm{D}$ and $\mathrm{B}-\mathrm{ALL}+\mathrm{A} 2 \mathrm{D}$ groups using ROS probe. The result showed that, indeed, the HSCs of B-ALL+A2D group had higher levels of intercellular ROS than those of the Ctrl+A2D group (Fig. $5 \mathrm{f}$ and g), which is consistent with increased cell proliferation and cell apoptosis in 


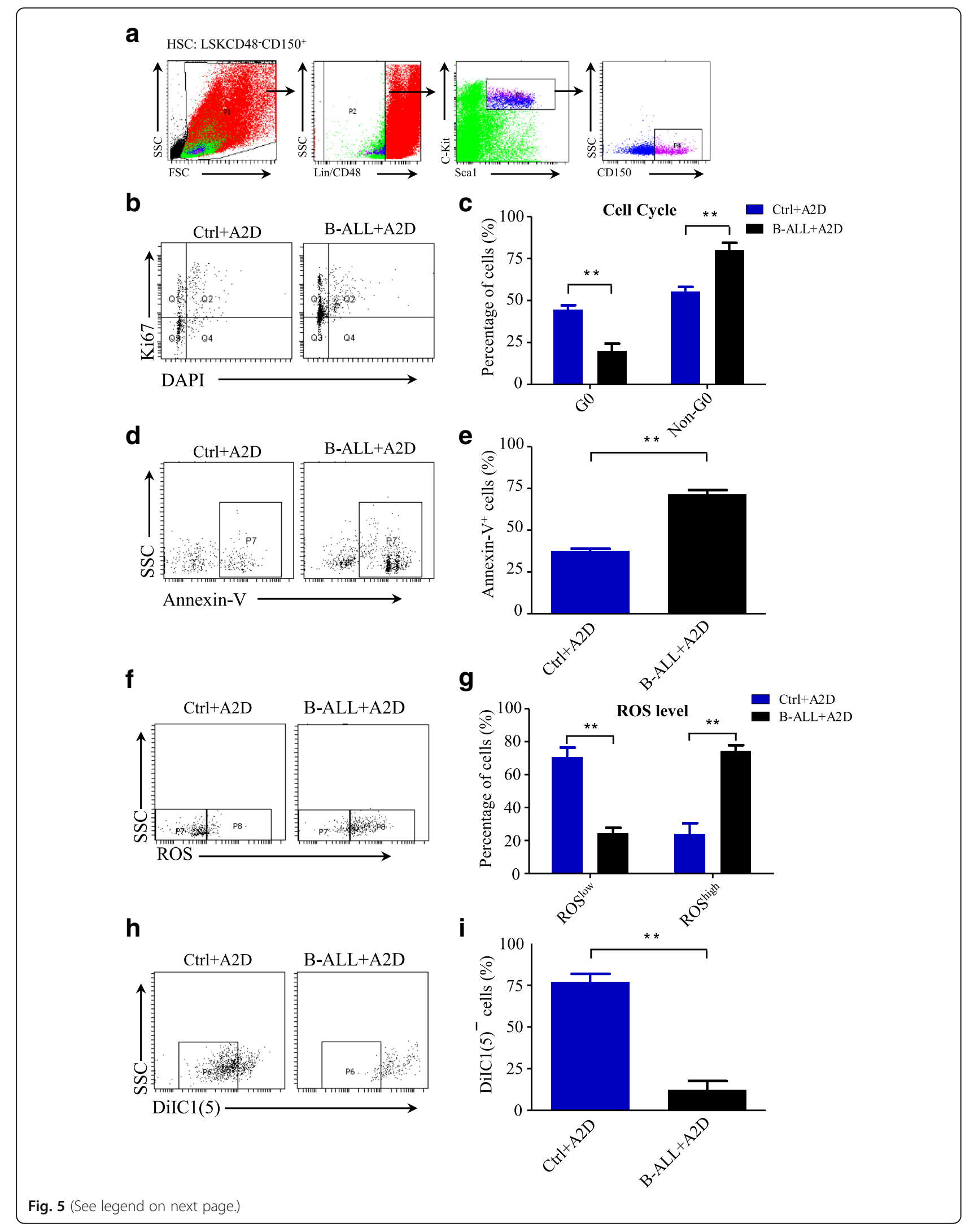




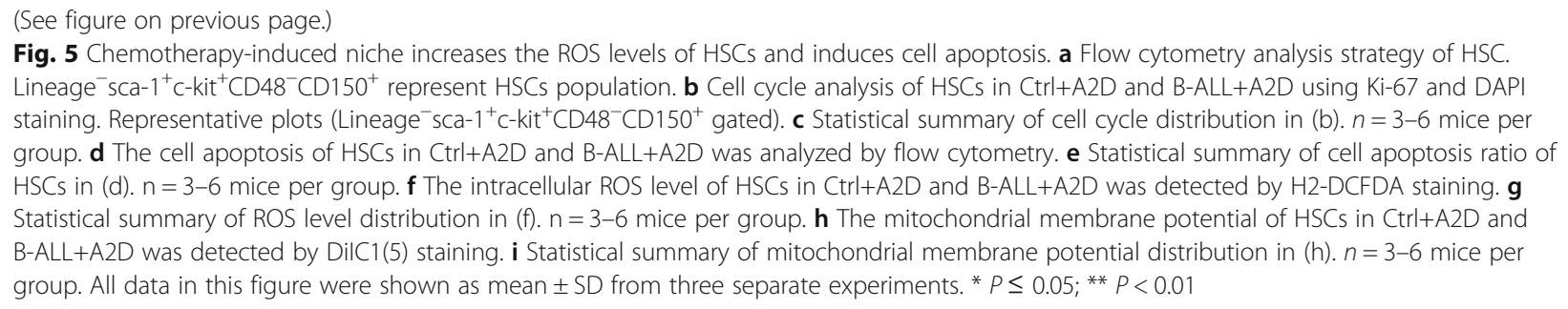

the B-ALL+A2D group. We also found that the ROS level was elevated in the HSCs of B-ALL after DNR treatment (Additional file 1: Figure S1c and d). Furthermore, we measured the mitochondrial membrane potential of the HSCs in the Ctrl+A2D and B-ALL+A2D groups using DilC-5 probe. Consistence with the intercellular ROS levels, the HSCs of B-ALL+A2D group had higher levels of mitochondrial membrane potential than those of the Ctrl+A2D group (Fig. 5h and i). These results indicated that the increasing of intercellular ROS levels and mitochondrial membrane potential at least contributes to the cell cycle and cell apoptosis of HSCs.

Altogether, these results suggest that chemotherapyinduced niche promotes HSCs entering cell cycle and increases cell apoptosis with increased the level of intracellular ROS and mitochondrial membrane potential, which may lead to the reduced hematopoietic reconstitution of HSCs.

\section{Discussion}

Chemotherapy has been shown to damage BM niche and cause myelosuppression, but the underlying mechanism remains controversial $[6,23,24]$. Here, we showed that a chemotherapy-induced niche in B-ALL perturbed the hematopoietic reconstitution of HSCs after transplantation, suggesting an indirect HSC damage mechanism mediated by BM niche. Our results implicated a fundamental role of the chemotherapy-induced niche on HSCs dysfunction and BM failure and demonstrated that chemotherapy agents can alternate the MSCs in the BM. These effects compromised the self-renewal capability of the HSCs. We also suggested that the decrease of cytokines from the MSCs and the increased level intracellular ROS in HSCs contribute to the perturbed hematopoietic reconstitution of HSCs after chemotherapy.

Various cytokines mediate the interaction between HSCs and niche components. Leptin receptor positive perivascular $\left(\mathrm{LepR}^{+}\right)$cells and Nestin ${ }^{+}$cells both express and secrete SCF, which is required for the maintenance of HSCs in the bone marrow [10, 11]. CXCL12 derived from mesenchymal progenitors is a niche factor that has been shown to regulate HSC functions, such as retention, quiescence and the ability to induce multilineage reconstitution $[25,26]$. Tie2-expressing HSCs situate closely with ANGPT-1-expressing stromal cells, and these interactions have been shown to enhance the adhesion of HSCs to osteoblastic cells through an upregulation of integrin $\beta 1$ [27]. Furthermore, angiogenin plays a non-cell-autonomous role in the regulation of hematopoiesis by simultaneously preserving the stemness of hematopoietic stem cells and progenitor cells and promoting the proliferation of lineage-committed myeloid-restricted progenitor cells [28]. Together, these studies highlighted the import roles of different cytokines in BM niche and HSCs maintenance. Here, we showed that these cytokines decreased in the chemotherapy-induced MSCs, and this decrease suggests an alternative myelosuppression mechanism for the maintenance of chemotherapycompromised HSCs maintenance in a "damaged niche."

Moreover, HSCs and their niche can be affected by diverse sources of stress, including oxidation, hypoxia, radiation, and chemotherapy, which disrupt HSC homeostasis and regeneration [29, 30]. Our results indicated that chemotherapy-induced niche facilitates the entry of quiescent HSCs into cell cycle and increases cell apoptosis by increasing the level of intracellular ROS and mitochondrial membrane potential, which may lead to the reduced hematopoietic reconstitution of HSCs. These results also suggest a potential indirect mechanism of chemotherapy-induced HSCs failure mediated by MSCs dysfunction. Meanwhile, our results implicated that the chemotherapy-damaged BM niche is not receptive to transplanted HSCs and thus may require the further assistance of HSCs-protective agents [31, 32]. Thus, these HSCs-protective agents may prevent long-term bone marrow failure and could provide a therapeutic option to increase BM function and decrease myelosuppression in chemotherapy-treated patients.

\section{Conclusion}

We report that chemotherapy-induced BM niche perturbs hematopoietic reconstitution through increasing the level of intracellular ROS and inducing the cell apoptosis of HSCs, possibly mediated by reduced cytokines in the niche. 


\section{Additional file}

Additional file 1: Figure S1. Chemotherapy-induced niche contributes to the increase of ROS and apoptosis in HSCs. (a) HSCs apoptosis in Ctrl +DNR and B-ALL+DNR was analyzed by flow cytometry. (b) Statistical summary of apoptotic cell ratio of HSCs in (a). $n=4-8$ mice per group. (c) The intracellular ROS level of HSCs in Ctrl+DNR and B-ALL+DNR was detected by H2-DCFDA staining. (d) Statistical summary of ROS level distribution in (c). $n=4-8$ mice per group. (PPTX $111 \mathrm{~kb}$ )

\section{Abbreviations}

ANGPT-1: Angiopoietin-1; B-ALL: B-cell acute lymphoblastic leukemia; CXCL12: C-X-C motif ligand 12; HSCs: Hematopoietic stem cells; IL7: Interleukin-7; MSCs: Mesenchymal stem cells; ROS: Reactive oxygen species; SCF: Stem cell factor; VCAM-1: Vascular cell adhesion molecule-1

\section{Acknowledgements}

We thank Jian-Min Zhu, Ying An (Shanghai Children's Medical Center, Shanghai Jiao Tong University School of Medicine) for excellent technical support.

\section{Funding}

This work is supported by the National Key R\&D Program of China, Stem Cell and Translation Research (NO. 2016 YFA0102000 to C.-W.D.) and the National Natural Science Foundation of China (NO. 81570121 to C.-W.D.; NO. 31530017 and 81372349 to B.-B.Z: NO 81500112 to H.L.) and The Science and Technology Commission of Pudong District, Shanghai Municipality (NO. PKJ2015-Y04 to C.-W.D.) and by Innovation Program of Shanghai Municipal Education Commission (NO. $15 Z Z 252$ to C.-W.D.)

\section{Availability of data and materials}

All data generated or analyzed for this study are included in this published article.

\section{Authors' contributions}

TC, ZBBS and DCW: designed the study, performed experiments (TC), analyzed and interpreted data, and wrote the paper; $\mathrm{LMH}, \mathrm{CYL}$ and SHY performed experiments, analyzed and interpreted data, LSL, ZWW, ZMY, LH FW and ZWJ performed experiments; LAB, TZH and HDL discussed results and contributed to data interpretation. All authors read and approved the final manuscript.

\section{Ethics approval}

All studies on animals were performed after approval by the Ethics Committee of Shanghai Children's Medical Center, in compliance with Guidelines for the Use and Care of Small Laboratory Animals.

\section{Consent for publication}

Not applicable.

\section{Competing interests}

The authors declare that they have no competing interests.

\section{Publisher's Note}

Springer Nature remains neutral with regard to jurisdictional claims in published maps and institutional affiliations.

\footnotetext{
Author details

${ }^{1}$ Key Laboratory of Pediatric Hematology and Oncology Ministry of Health and Pediatric Translational Medicine Institute, Shanghai Children's Medical Center, Shanghai Collaborative Innovation Center for Translational Medicine and Department of Pharmacology and Chemical Biology, Shanghai Jiao Tong University School of medicine (SJTU-SM), Shanghai 200025, China. 2Department of Hematology, Tongji Hospital, Tongji University School of Medicine, Shanghai 200092, People's Republic of China. ${ }^{3}$ Key Laboratory of Cell Differentiation and Apoptosis of National Ministry of Education, Department of Pathophysiology, SJTU-SM, Shanghai 200025, China.
}

Received: 7 May 2018 Accepted: 25 July 2018

Published online: 29 August 2018

\section{References}

1. Orkin SH, Zon LI. Hematopoiesis: an evolving paradigm for stem cell biology. Cell. 2008;132(4):631-44.

2. Doulatov S, Notta F, Laurenti E, Dick JE. Hematopoiesis: a human perspective. Cell Stem Cell. 2012;10(2):120-36.

3. Cabezas-Wallscheid N, Buettner F, Sommerkamp P, Klimmeck D, Ladel L, Thalheimer FB, Pastor-Flores D, Roma LP, Renders S, Zeisberger P, et al. Vitamin A-retinoic acid signaling regulates hematopoietic stem cell dormancy. Cell. 2017;169(5):807-23. e819

4. Haas R, Mohle R, Fruhauf S, Goldschmidt H, Witt B, Flentje M, Wannenmacher M, Hunstein W. Patient characteristics associated with successful mobilizing and autografting of peripheral blood progenitor cells in malignant lymphoma. Blood. 1994;83(12):3787-94.

5. Elmaagacli AH, Koldehoff $M$, Steckel NK, Trenschel $R$, Ottinger $H$, Beelen DW. Cytochrome P450 2C19 loss-of-function polymorphism is associated with an increased treatment-related mortality in patients undergoing allogeneic transplantation. Bone Marrow Transplant. 2007:40(7):659-64.

6. Wang Y, Probin V, Zhou D. Cancer therapy-induced residual bone marrow injury-mechanisms of induction and implication for therapy. Curr Cancer Ther Rev. 2006;2(3):271-9.

7. To LB, Levesque JP, Herbert KE. How I treat patients who mobilize hematopoietic stem cells poorly. Blood. 2011;118(17):4530-40.

8. Crane GM, Jeffery E, Morrison SJ. Adult haematopoietic stem cell niches. Nat Rev Immunol. 2017;17(9):573-90.

9. Gao X, Xu C, Asada N, Frenette PS. The hematopoietic stem cell niche: from embryo to adult. Development. 2018;145(2). https://doi.org/10.1242/dev. 139691.

10. Kunisaki Y, Bruns I, Scheiermann C, Ahmed J, Pinho S, Zhang D, Mizoguchi T, Wei Q, Lucas D, Ito K, et al. Arteriolar niches maintain haematopoietic stem cell quiescence. Nature. 2013;502(7473):637-43.

11. Acar M, Kocherlakota KS, Murphy MM, Peyer JG, Oguro H, Inra CN, Jaiyeola C, Zhao Z, Luby-Phelps K, Morrison SJ. Deep imaging of bone marrow shows non-dividing stem cells are mainly perisinusoidal. Nature. 2015; 526(7571):126-30

12. Quasthoff $S$, Hartung HP. Chemotherapy-induced peripheral neuropathy. J Neurol. 2002;249(1):9-17.

13. Crawford J, Dale DC, Lyman GH. Chemotherapy-induced neutropenia: risks, consequences, and new directions for its management. Cancer. 2004;100(2): 228-37.

14. Pui $\mathrm{CH}$, Yang JJ, Hunger SP, Pieters $\mathrm{R}$, Schrappe $\mathrm{M}$, Biondi A, Vora A Baruchel A, Silverman LB, Schmiegelow K, et al. Childhood acute lymphoblastic leukemia: progress through collaboration. J Clin Oncol. 2015; 33(27):2938-48.

15. Duan CW, Shi J, Chen J, Wang B, Yu YH, Qin X, Zhou XC, Cai YJ, Li ZQ, Zhang $F$, et al. Leukemia propagating cells rebuild an evolving niche in response to therapy. Cancer Cell. 2014;25(6):778-93.

16. Sugihara E, Shimizu T, Kojima K, Onishi N, Kai K, Ishizawa J, Nagata K, Hashimoto N, Honda H, Kanno M, et al. Ink4a and Arf are crucial factors in the determination of the cell of origin and the therapeutic sensitivity of Myc-induced mouse lymphoid tumor. Oncogene. 2012; 31(23):2849-61.

17. Kusumbe AP, Ramasamy SK, Starsichova A, Adams RH. Sample preparation for high-resolution 3D confocal imaging of mouse skeletal tissue. Nat Protoc. 2015;10(12):1904-14.

18. Pinho S, Lacombe J, Hanoun M, Mizoguchi T, Bruns I, Kunisaki Y, Frenette PS. PDGFRalpha and CD51 mark human nestin+ sphere-forming mesenchymal stem cells capable of hematopoietic progenitor cell expansion. J Exp Med. 2013;210(7):1351-67.

19. Wilson A, Laurenti $E$, Oser $G$, van der Wath RC, Blanco-Bose W, Jaworski M, Offner S, Dunant CF, Eshkind L, Bockamp E, et al. Hematopoietic stem cells reversibly switch from dormancy to self-renewal during homeostasis and repair. Cell. 2008:135(6):1118-29.

20. Kiel MJ, Yilmaz OH, Iwashita T, Terhorst C, Morrison SJ. SLAM family receptors distinguish hematopoietic stem and progenitor cells and revea endothelial niches for stem cells. Cell. 2005;121(7):1109-21.

21. Ray PD, Huang BW, Tsuji Y. Reactive oxygen species (ROS) homeostasis and redox regulation in cellular signaling. Cell Signal. 2012;24(5):981-90. 
22. Ito K, Hirao A, Arai F, Takubo K, Matsuoka S, Miyamoto K, Ohmura M, Naka K, Hosokawa K, lkeda Y, et al. Reactive oxygen species act through p38 MAPK to limit the lifespan of hematopoietic stem cells. Nat Med. 2006;12(4):446-51.

23. Kemp K, Morse R, Wexler S, Cox C, Mallam E, Hows J, Donaldson C.

Chemotherapy-induced mesenchymal stem cell damage in patients with hematological malignancy. Ann Hematol. 2010;89(7):701-13.

24. Lucas D, Scheiermann C, Chow A, Kunisaki Y, Bruns I, Barrick C, Tessarollo L, Frenette PS. Chemotherapy-induced bone marrow nerve injury impairs hematopoietic regeneration. Nat Med. 2013;19(6):695-703.

25. Ding L, Morrison SJ. Haematopoietic stem cells and early lymphoid progenitors occupy distinct bone marrow niches. Nature. 2013;495(7440): 231-5.

26. Greenbaum A, Hsu YM, Day RB, Schuettpelz LG, Christopher MJ, Borgerding $\mathrm{JN}$, Nagasawa T, Link DC. CXCL12 in early mesenchymal progenitors is required for haematopoietic stem-cell maintenance. Nature. 2013;495(7440): 227-30.

27. Arai F, Hirao A, Ohmura M, Sato H, Matsuoka S, Takubo K, Ito K, Koh GY, Suda T. Tie2/angiopoietin-1 signaling regulates hematopoietic stem cell quiescence in the bone marrow niche. Cell. 2004;118(2):149-61.

28. Goncalves KA, Silberstein L, Li S, Severe N, Hu MG, Yang H, Scadden DT, Hu GF. Angiogenin promotes hematopoietic regeneration by dichotomously regulating quiescence of stem and progenitor cells. Cell. 2016;166(4):894-906.

29. Signer RA, Morrison SJ. Mechanisms that regulate stem cell aging and life span. Cell Stem Cell. 2013;12(2):152-65.

30. Suda T, Takubo K, Semenza GL. Metabolic regulation of hematopoietic stem cells in the hypoxic niche. Cell Stem Cell. 2011:9(4):298-310.

31. Bisi JE, Sorrentino JA, Roberts PJ, Tavares FX, Strum JC. Preclinical characterization of G1T28: a novel CDK4/6 inhibitor for reduction of chemotherapy-induced myelosuppression. Mol Cancer Ther. 2016;15(5):783-93.

32. He S, Roberts PJ, Sorrentino JA, Bisi JE, Storrie-White H, Tiessen RG, Makhuli KM, Wargin WA, Tadema H, van Hoogdalem EJ, et al. Transient CDK4/6 inhibition protects hematopoietic stem cells from chemotherapy-induced exhaustion. Sci Transl Med. 2017;9(387). https://doi.org/10.1126/scitransImed. aal3986.

Ready to submit your research? Choose BMC and benefit from:

- fast, convenient online submission

- thorough peer review by experienced researchers in your field

- rapid publication on acceptance

- support for research data, including large and complex data types

- gold Open Access which fosters wider collaboration and increased citations

- maximum visibility for your research: over $100 \mathrm{M}$ website views per year

At $\mathrm{BMC}$, research is always in progress.

Learn more biomedcentral.com/submissions 